\title{
Une envie de peindre : les aquarelles inédites de Julius Euting
}

Julia Bischoff

\section{OpenEdition}

1 Journals

Édition électronique

URL : http://journals.openedition.org/rbnu/320

DOI : 10.4000/rbnu.320

ISSN : 2679-6104

Éditeur

Bibliothèque nationale et universitaire de Strasbourg

\section{Édition imprimée}

Date de publication : 23 mai 2018

Pagination : 112-119

ISSN : 2109-2761

\section{Référence électronique}

Julia Bischoff, « Une envie de peindre : les aquarelles inédites de Julius Euting », La Revue de la BNU [En ligne], 17 | 2018, mis en ligne le 05 septembre 2018, consulté le 10 décembre 2020. URL : http:// journals.openedition.org/rbnu/320 ; DOI : https://doi.org/10.4000/rbnu.320

\section{(c) (i) (9)}

La Revue de la BNU est mise à disposition selon les termes de la Licence Creative Commons Attribution - Pas d'Utilisation Commerciale - Partage dans les Mêmes Conditions 4.0 International. 
VARIA

\section{Une envie de peindre: les aquarelles inédites de Julius Euting}

PAR JULIA BISCHOFF

(RÉVISION FRANCCAISE CLAUDE LORENTZ)

Julius Euting assis au milieu d'un camp d'expédition proche de Pétra,

photographe inconnu, 1898 , succession Julius Euting, $\mathrm{n}^{\circ}$ inv. :

N 3.08, F 213, Nr. 2 (coll. Archives de la Ville de Freudenstadt)

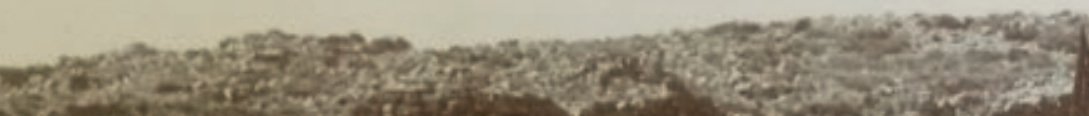


Assis sur un tabouret et penché sur ses outils de dessin et d'écriture, étalés sur une table pliante, le bibliothécaire et orientaliste Julius Euting (1839-1913) est affairé, immergé dans ses notes, au milieu d'un camp d'expédition dans le désert jordanien (voir ill. ci-contre).

En cet instant, saisi par un photographe resté inconnu, Euting participe à l'expédition scientifique de 1898 dans la ville antique de Pétra, la capitale de l'ancien royaume nabatéen - expédition initiée par les orientalistes Rudolf Ernst Brünnow (1858-1917) et Alfred von Domaszewski (1856-1927). Son concours avait été sollicité eu égard à ses compétences philologiques et épigraphiques dans le domaine des langues sémitiques anciennes ${ }^{1}$.

Premier bibliothécaire à la Kaiserliche Universitäts- und Landesbibliothek zu Strassburg (KULBS, l'ancêtre de la BNU) depuis 1872 (il en deviendra le directeur de 1900 à 1909), philologue, spécialiste des langues anciennes du Proche-Orient, professeur honoraire à la Kaiser-WilhelmsUniversität de Strasbourg, Euting, un orientaliste reconnu, n'en était pas à sa première expédition. Ses recherches épigraphiques et sa quête de documents originaux pour la KULBS l'avaient mené de Constantinople au désert arabique en passant par l'Italie, la Grèce, l'Europe centrale, la Tunisie et l'Anatolie ${ }^{2}$.

Si Euting était bibliothécaire par profession, chercheur-orientaliste par vocation, il était également animé d'une autre passion : le dessin et la peinture, notamment l'aquarelle. Durant ses voyages et expéditions, il avait pris l'habitude de décrire les événements et de dessiner méticuleusement les paysages, personnes, plantes et même les animaux qu'il rencontrait ou croisait. Un nombre étonnant de vingt-trois journaux de voyages contenant des descriptions détaillées aussi bien que des dessins au crayon et à la plume nous sont ainsi parvenus ${ }^{3}$. Il existe de surcroît vingt-cinq cahiers de dessins et d'aquarelles, ainsi qu'une trentaine d'aquarelles conservées individuellement, sans cahier, dans des collections publiques et privées en France et en Allemagne ${ }^{4}$. Ces chiffres témoignent d'une productivité artistique qui dépasse le simple passe-temps d'un amateur. Pour Euting, l'expression artistique a joué un rôle majeur durant ses expéditions et dans sa vie privée. Qui était donc ce personnage ? Qu'a-t-il peint pendant ses voyages en Orient ? Enfin, quelle est la particularité, l'originalité, de ses dessins et peintures?

\section{Un don inné}

Julius Euting est né le 11 juillet 1839 dans une famille bourgeoise de Stuttgart. Son père, Franz Jakob Euting (1812-1879), occupait un poste de fonctionnaire d'État au ministère de l'Intérieur du Wurtemberg ${ }^{5}$. Il était très attentif à la bonne éducation de son fils Julius et de ses six frères et sœurs. Le jeune Julius a ainsi fréquenté l'un des plus prestigieux lycées de la ville de Stuttgart, l'Eberhard-Ludwigs-Gymnasium. À l'âge de 14 ans, il poursuivit sa formation dans le séminaire de théologie protestante de Blaubeuren, une petite ville proche d'Ulm.

Son père envisageait pour son second fils Julius une carrière ecclésiastique. Il en posa les jalons en l'inscrivant, à l'âge de 17 ans, dans l'établissement le plus reconnu du royaume du Wurtemberg, le Stift de Tübingen, pour des études de théologie protestante. L'intérêt de l'étudiant s'orienta cependant davantage vers l'étude des langues sémitiques comme l'hébreu, le phénicien, l'araméen et l'arabe, que vers la théologie protestante. En 1862, il acheva son cycle d'études avec une thèse de doctorat sur la troisième sourate $\mathrm{du}$ Coran ${ }^{6}$.

Euting travailla ensuite comme précepteur au sein d'une famille de la noblesse, près de la petite ville de Babstadt dans le Wurtemberg ${ }^{7}$. En 1866, on lui proposa un poste de bibliothécaire à Tübingen, qu'il occupa pendant six ans. À ce moment-là, il avait déjà commencé à pratiquer la peinture à l'eau dont il avait découvert la technique au cours d'un voyage d'études à Londres ${ }^{8}$. Dès son très jeune âge, Julius Euting avait dessiné des bâtiments, des églises et des paysages au crayon fin. Son biographe Hans Graner mentionne que le dessin et la peinture à l'eau auraient "pendant toute sa vie » fait partie « de ses occupations préférées » ${ }^{9}$. Par ailleurs, Graner raconte qu'Euting aurait été d'une « humeur particulièrement mauvaise » quand il rentrait d'une randonnée, d'une excursion ou d'un voyage sans avoir pu capturer suffisamment d'impressions sur papier. Le dessin lui était un moyen indispensable d'exprimer les moments vécus et il était animé d'un besoin irrésistible d'esquisser les paysages qu'il voyait autour de lui ${ }^{10}$.

Les fonds de la Bibliothèque nationale et universitaire de Strasbourg contiennent à ce propos de remarquables exemples de dessins réalisés pendant la 
jeunesse de Julius Euting à Stuttgart. Ils montrent des immeubles de la capitale de Wurtemberg, ainsi que des dessins exécutés lors de randonnées et sorties en famille ${ }^{11}$.

À la fin de la guerre franco-prussienne de 1870, Julius Euting était toujours bibliothécaire à Tübingen. L'issue de la guerre se solda par l'annexion de l'AlsaceLorraine à l'Empire allemand nouvellement créé et, de fait, par la création de nouveaux postes d'État dans les régions conquises. Les postes des institutions publiques, notamment universitaires, furent restructurés et redistribués principalement à des fonctionnaires d'origine allemande ${ }^{12}$, non sans générer des tensions avec la population locale alsacienne et lorraine.

Suite à l'incendie et à la destruction totale de la bibliothèque de la ville, la bibliothèque du Temple Neuf, en août 1870, une nouvelle bibliothèque, régionale et universitaire, fut fondée à Strasbourg, la KULBS déjà citée ${ }^{13}$, sous l'impulsion et l'égide de Karl August Barack (1827-1900) qui en devint le premier directeur. En 1872, Barack fit appel à Euting et le chargea, entre autres missions, de développer des fonds orientalistes. Barack profita par ailleurs largement de l'expérience de bibliothécaire de son nouvel employé, en faisant par exemple adopter pour la KULBS un modèle de classement systématique largement inspiré de celui que ce dernier avait déjà établi à Tübingen ${ }^{14}$.

En dehors de son activité scientifique et de bibliothécaire, il est important de noter que Julius Euting se faisait également remarquer par son engagement social pour les habitants de Strasbourg, ainsi que par sa contribution au développement touristique de l'Alsace ${ }^{15}$. Il contribua à la valorisation du massif vosgien et publia plusieurs guides touristiques destinés à faire connaître la région ${ }^{16}$. Ses randonnées et expéditions furent autant d'occasions pour lui de croquer et de dessiner les paysages qui l'entouraient.

Euting resta à Strasbourg jusqu'à la fin de sa vie. En 1900, il devint directeur de la KULBS ${ }^{17}$. Avant cela, il avait entrepris plusieurs grands voyages, comme notamment une expédition en Syrie et en Arabie du Nord avec son compagnon français Charles Huber (1847-1884) ${ }^{18}$ en 1883-1884, mais aussi en Syrie du Nord entre 1889 et 1890 pour les fouilles archéologiques de Zincirli (Sam'al) avec le chercheur autrichien Felix Ritter von Luschan (1854-1924), à Pétra et dans le désert jordanien enfin, en 1898, avec les orientalistes Brünnow et von Domaszewski.
En 1913, son collègue orientaliste de l'Université de Strasbourg, Theodor Nöldeke (18361930), faisait, dans la notice nécrologique d'Euting, l'éloge de sa forte capacité visuelle. Il écrivait que ce dernier était capable de mémoriser les formes et les lignes des paysages, des inscriptions et des lettres sémitiques qu'il pouvait encore redessiner correctement quelques jours après les avoir vues. Ce « don inné pour le dessin » aurait été d'un incroyable avantage pour la science, car la plupart des orientalistes précédents n'auraient pas été capables de reproduire correctement les inscriptions ${ }^{19}$. Mais Nöldeke allait encore plus loin en affirmant qu'il y avait là trace d'aspirations professionnelles non réalisées de Julius Euting. Dans sa notice, il confirmait que celui-ci aurait avant tout « préféré devenir peintre ${ }^{20}$.

Ce désir ne s'est jamais réalisé. Son « don inné " resta sans la formation artistique spécialisée qui aurait été nécessaire afin de le raffiner et de le perfectionner. Néanmoins, malgré son caractère dilettante, l'œuvre picturale d'Euting fait preuve d'une maîtrise exceptionnelle des techniques et d'une conscience aiguë de la composition esthétique. Son activité artistique étant principalement liée à sa mobilité touristique et scientifique dans les Vosges comme au Moyen-Orient, les aquarelles et dessins réalisés représentent des motifs de paysages, de plantes ou de personnages et objets qu'il a rencontrés. Dans les fonds de la BNU se trouve notamment un Recueil d'aquarelles de paysages arabes ${ }^{21}$ qui contient des trésors inédits de représentations de paysages et sites moyen-orientaux du dernier tiers du $19^{\mathrm{e}}$ siècle. Il s'agit d'un cahier de voyage dans lequel Euting avait réalisé des dessins au crayon ainsi que des aquarelles entre 1884 et 1910.

\section{Les aquarelles de Mar Saba}

Si l'on revient à la photographie retrouvée de Julius Euting au milieu du camp d'expédition, évoquée au début de cet article, les aquarelles réalisées pendant son voyage en Jordanie en 1898 avec ses collègues Rudolf Ernst Brünnow et Alfred von Domaszewski nous intéressent tout particulièrement.

Pour Euting, cette expédition commença le 23 janvier 1898 à la gare de Strasbourg, où il avait 

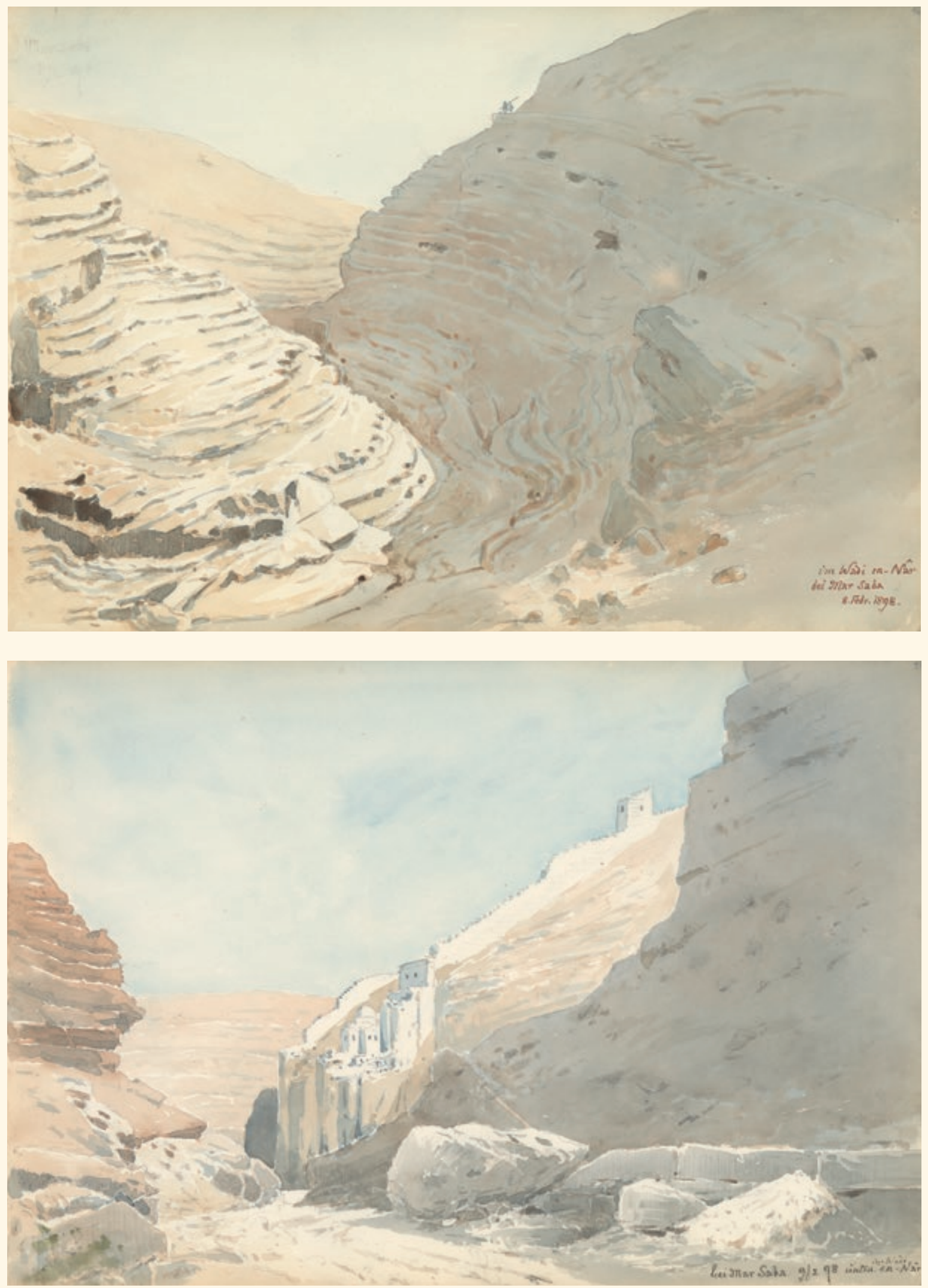

(Haut) Julius Euting, «Im Wadi en-Nâr bei Mar Saba 8.Febr.1898. », couleurs à l'eau et crayon sur papier vélin, 250 × 345 mm, 1898, in Recueil d'aquarelles de paysages arabes, MS.7.094, fol. 22 (coll. BNU)

(Bas) Julius Euting, « Bei Mar Saba 9/2 98 unten im Wadi en-Nâr », couleurs à l'eau et crayon sur papier vélin, 250 × 345 mm, 1898, in Recueil d'aquarelles de paysages arabes, MS.7.094, fol. 23 (coll. BNU) 

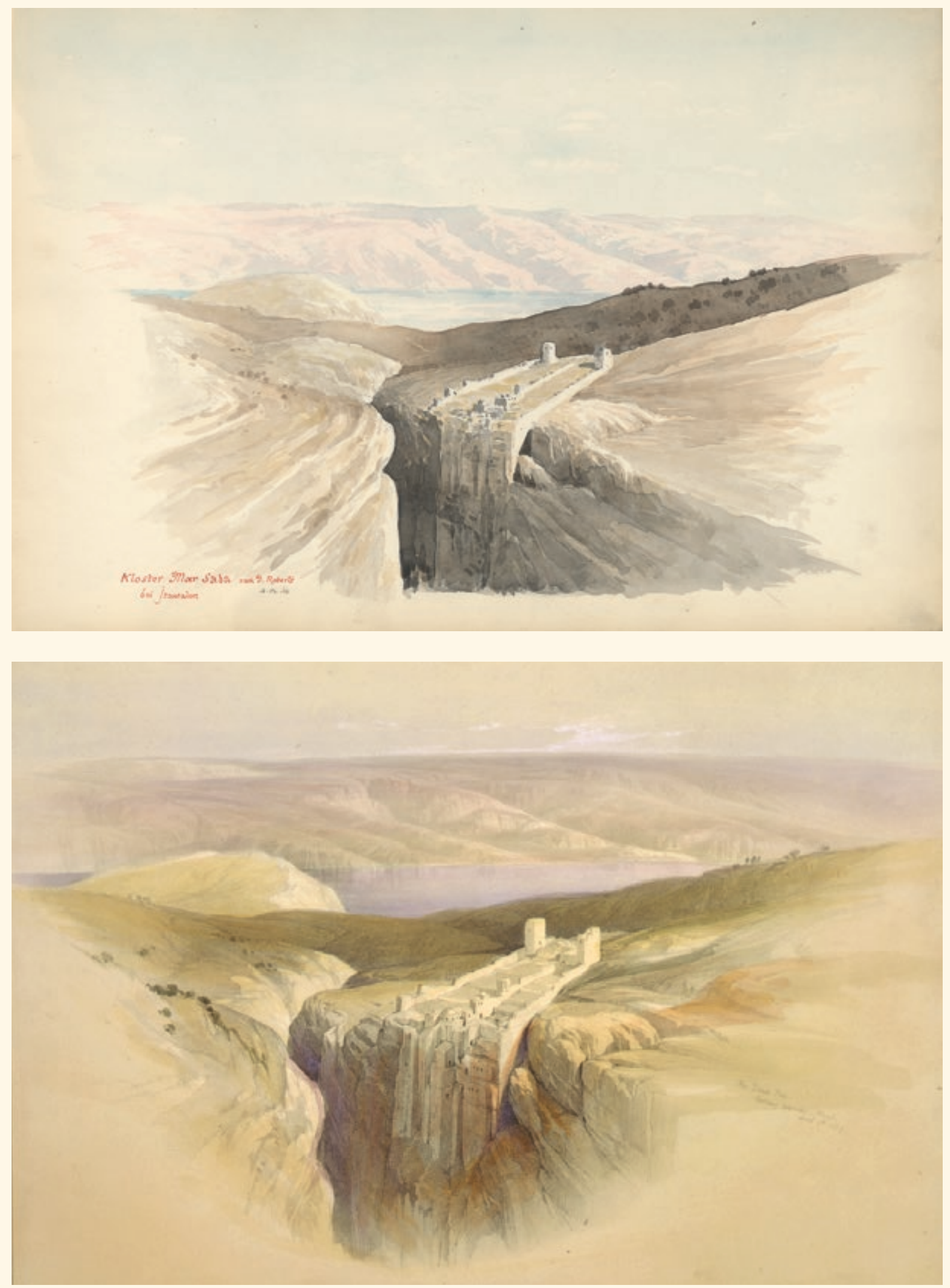

(Haut) Julius Euting, « Kloster Mar Saba bei Jerusalem nach D. Roberts 4.12.10 », couleurs à l'eau et crayon sur papier vélin, 250 × 345 mm, 1910, in Recueil d'aquarelles de paysages arabes, MS.7.094, fol. 33 (coll. BNU).

(Bas) David Roberts, «The Dead Sea, looking towards Moab. April $4^{\text {th }} 1839$ », lithographie, Louis Haghe (graveur), 1843, 233 x 333mm, in The Holy Land, Syria, Idumea, Arabia, Egypt and Nubia / from drawings made on the spot by David Roberts..., London, 1842-1845, v. 2, pts. 7-8, p. 6 (coll. Library of Congress) ${ }^{29}$. 
pris le train pour Bâle en Suisse. Il écrivait ainsi dans son journal de voyage : « Je me suis réveillé à 3 heures $\mathrm{du}$ matin [...] J'avais trois bagages avec moi, mon nouveau sac à main, mon sac à dos et mon rouleau de tôle (Blechrolle) ${ }^{22}$ ". Il prit ensuite le bateau à Gênes, en Italie, pour traverser la Méditerranée et arriver dans le port de Jaffa en Palestine. Il emprunta la direction sud-est, passa quelques jours à Jérusalem ainsi qu'au monastère de Mar Saba proche de la mer Morte. Fondé probablement au $5^{\mathrm{e}}$ siècle par le saint Sabas le Sanctifié, Mar Saba compte parmi les plus anciens monastères au monde ${ }^{23}$.

Le 8 février 1898, Euting et son expédition arrivèrent au Wadi en-Nâr, une vallée dans les environs de Mar Saba. Dans son journal de voyage, Euting a noté explicitement que ce soir-là, il avait pris le temps de dessiner ${ }^{24}$. Dans le recueil d'aquarelles, on en découvre effectivement une datée de ce jour et représentant le paysage du Wadi en-Nâr (voir ill. p. 115 en haut).

Euting utilisait en général, pour la réalisation de ses aquarelles, la même technique : il commençait à définir les contours et les lignes de composition de ses œuvres avec des ébauches fines au crayon doux. Ensuite, il rajoutait des couleurs à l'eau pour exprimer l'ambiance générale, les détails et les effets d'ombres. Les couleurs à l'eau utilisées gardaient toujours leur aspect transparent et clair car il s'abstenait d'y adjoindre quelque additif opaque ${ }^{25}$. Les papiers utilisés provenaient de cahiers de peinture de bonne qualité $^{26}$. Les vélins, en particulier, pouvaient absorber l'eau très rapidement et ainsi fixer parfaitement les pigments sur leur surface.

Dans cette aquarelle du Wadi en-Nâr, Euting laisse transparaître la couleur beige clair du papier comme effet de lumière sur les falaises de la vallée. Le Wadi en-Nâr est sillonné par différentes strates de rochers et semble raconter l'histoire d'une ancienne et puissante rivière. L'aquarelliste capte le mouvement souple des rochers. Il crée une composition triangulaire entre les deux falaises qui dirige le regard du spectateur et suscite la curiosité de ce qui se trouve derrière la grande falaise de droite. Cet effet est amplifié par les deux petits personnages indiqués sur un chemin étroit en haut de la falaise. Le spectateur a automatiquement tendance à s'imaginer la vue de ces deux voyageurs qui viennent de dépasser la courbe de la vallée.
Cette aquarelle représente aussi la joie anticipée de Julius Euting pendant la traversée du Wadi en-Nâr. Il savait que la route mènerait directement au pied du monastère de Mar Saba, un lieu qu'il avait attendu de découvrir depuis longtemps. Le lendemain, il écrivait dans son journal : «Mar Saba 9.2.98 [...] Je me suis réveillé à 6 heures [...] J'ai descendu le Wadi Nâr en dessinant [...] jusqu'au monastère de Mar Saba ${ }^{27}$.

L'issue de cette descente est représentée dans une nouvelle aquarelle qui montre une vue sur le monastère depuis le fond de la vallée (voir ill. p. 115 en bas). Entre les falaises qui se dressent des deux côtés de l'image, il y a un chemin positionné au milieu de la composition, qui mène au pied du monastère. Mar Saba est représenté comme une architecture impressionnante, dominant le rocher qu'il descend avec un long mur de fortification et un complexe de bâtiments qui apparaissent tous dans une couleur plus claire que leur environnement. L'intention de Julius Euting consiste ici à mettre en valeur l'architecture extraordinaire du monastère par rapport à la nature brute et au paysage aride tout autour. En réalité, cependant, le monastère est bien sûr construit avec les rochers de la région, dans des tons très proches de son environnement.

Avec des pigments bien dissous dans l'eau, Euting a créé une couche bleu clair de grande étendue sur l'arrière-plan. Les parties avec un peu plus de concentration de pigments évoquent naturellement des nuages. À partir de cette aquarelle, nous pouvons constater la bonne maitrise par Euting des différentes techniques de la peinture à l'eau. Sur certaines parties comme sur les rochers au bord du chemin à droite, on remarque des points de couleur rapprochés et précisément placés afin de créer les effets de réflexion du soleil. Dans les rochers de gauche, Euting superpose plusieurs couches picturales afin de créer des effets de transparence et de faire fondre les différentes couleurs appliquées.

Il est remarquable de noter que durant l'étape très courte à Mar Saba - l'expédition n'y resta que deux jours, sur le chemin vers Pétra -, Euting prit le temps de réaliser au total quatre aquarelles, alors qu'aucune ne fut réalisée à Pétra même. Arrivé au site archéologique nabatéen, sa production artistique céda manifestement la place aux analyses scientifiques et aux copies d'inscriptions qui remplissent dorénavant les pages de ses journaux de voyages. Le savant reprit 
ses crayons et pinceaux sur le chemin du retour. La peinture et le dessin restaient ainsi un luxe, un passetemps important, mais qui passait au second plan par rapport à son travail scientifique.

Bibliothécaire, savant, chercheur-orientaliste, Julius Euting l'était assurément. Mais pouvonsnous également, aujourd'hui, lui attribuer le titre de peintre ? Cette dimension artistique, voire ce rêve d'être reconnu en tant que peintre, l'accompagnèrent à l'évidence tout au long de sa vie. S'il n'a jamais publié ni vendu ses réalisations, il y consacra néanmoins un soin tout particulier, en étiquetant méthodiquement ses cahiers de peinture et en les conservant jusqu'à la fin de sa vie.

En 1910, environ deux ans avant sa mort, le motif du monastère de Mar Saba qu'il avait visité douze ans auparavant, lors de l'expédition de Pétra, réapparut dans son œuvre. Il nous semble ainsi évident aujourd'hui que cette image a joué un rôle particulier dans la perception par Euting du paysage oriental, en même temps qu'elle contribuait certainement au souvenir nostalgique du temps des expéditions et des aventures.

Une autre aquarelle (voir ill. p. 116 en haut) témoigne de la même finesse d'application des couches de couleurs fines et transparentes comme dans ses œuvres précédentes. Le ciel bleu clair ainsi que les montagnes roses à l'arrière-plan prêtent une légèreté à la composition et laissent découvrir la mer Morte derrière la vallée et les falaises du Wadi en-Nâr. Cependant, cette fois-ci, l'architecture de Mar Saba est représentée de manière plus dramatique que dans la version de 1898. Les bâtiments semblent en voie d'être engloutis par la gorge, et la vallée apparaît plus étroite, plus noire et dangereuse.

Julius Euting se référait incontestablement, dans cette œuvre tardive, à une lithographie qu'il avait connue depuis ses premières études de peinture à l'eau en 1866. Il s'agit de The Dead Sea, looking towards Moab (voir ill. p. 116 en bas), une lithographie tirée d'un tableau du peintre orientaliste anglais David Roberts (1796-1864). Ce dernier avait publié entre 1842 et 1845 une série de peintures orientalistes lithographiées par le graveur belge Louis Haghe (1806-1864) ${ }^{28}$, et qu'Euting avait vraisemblablement connues pendant ses recherches à Londres.
À la fin de sa vie, Euting a donc réalisé une copie de ce fameux motif orientaliste qu'il avait vu sur place et dessiné de manière plus réaliste quelques années auparavant. Elle témoigne de son inclination durable envers l'art pictural. Cet aspect du personnage d'Euting demeure un terrain d'étude encore largement ouvert à la recherche. Son œuvre offre en tout cas une vision intime et une représentation encore inédite de l'Orient à la fin du $19^{\mathrm{e}}$ siècle.

\section{ORIENTATIONS BIBLIOGRAPHIQUES}

- Bischoff, Julia, Unterwegs in Wald und Wüste. Studien zu den Tage- und Skizzenbüchern des schwäbischen Orientforschers Julius Euting, mémoire de fin d'études, master Histoire de l'art et muséologie à l'Université de Heidelberg et à l'École du Louvre (Paris), mars 2017

- Euting, Julius, Guide illustré de la Ville de Strasbourg et de la Cathédrale, Strasbourg, 1881 (sept éditions françaises, sept éditions anglaises, quatorze éditions allemandes. Titre allemand : Beschreibung der Stadt Straßburg und des Münsters. Titre anglais : A Descriptive Guide to the City of Strassburg and its Cathedral).

- Euting, Julius, Tagebuch einer Reise in Inner-Arabien, Erster Theil, Leiden, 1896

- Euting, Julius, Tagebuch einer Reise in Inner-Arabien, Zweiter Theil, Enno Littmann (éd.), Leiden, 1914

- Goer, Charis et Hoffmann, Michael (éd.), Der Deutschen Morgenland. Bilder des Orients in der deutschen Literatur und Kultur von 1770 bis 1850, München, 2008

- Mangold, Sabine, Eine « weltbürgerliche Wissenschaft ». Die deutsche Orientalistik im 19. Jahrhundert (Beiträge zur Universitäts- und Wissenschaftsgeschichte, Band 11), Rüdiger vom Bruch et Eckart Henning (éd.), Stuttgart, 2004

- Marchand, Suzanne L., German Orientalism in the Age of Empire. Religion, Race and Scholarship, New York, 2009

- Mündel, Curt, Die Vogesen. Reisehandbuch für Elsass-Lothringen und angrenzende Gebiete, unter Mitwirkung von Prof. Dr. Julius Euting und Prof. Dr. Otto Bechstein, Strasbourg, 1881 (première édition), 1906 (onzième édition)

\section{NOTES}

1- Brünnow, Rudolf-Ernst, Domaszewski, Alfred von, Kunze, Max Friedrich et Euting, Julius, Die Provincia Arabia auf Grund zweier in den Jahren 1897 und 1898 unternommenen Reisen und der Berichte früherer Reisender, Strassburg, K. J. Trübner, 1904-1909, 3 vol.

2- 1867 : voyage à Istanbul ; 1869 : voyage à travers l'Italie jusqu'à Tunis ; 1870 : voyage en Turquie ; 1874 : voyage à Londres pour la conférence internationale des orientalistes ; 1881 : conférence internationale des orientalistes à Berlin ; 1883-1884 : grande expédition en Syrie et en Arabie du Nord avec Charles Huber ; 1886 : conférence internationale des orientalistes à Vienne (Autriche) ; 1889 : expédition scientifique en Égypte et dans la péninsule du Sinaï ; 1889 : conférence internationale des 
orientalistes à Stockholm et Christiana ; 1889-1890 : grande expédition scientifique en Syrie du Nord (fouilles archéologiques de Zincirli/Sam'al) avec Felix von Luschan ; 1898 : expédition scientifique en Jordanie (Pétra) avec Rudolf Ernst Brünnow et Alfred von Domaszewski ; 1903 : expédition en Syrie et en Égypte ; 1905 : conférence internationale des orientalistes à Alger et Tunis.

3- Ils sont conservés à la Bibliothèque universitaire de Tübingen et sont accessibles en ligne par un lien permanent : http://idb.ub.unituebingen.de/diglit/Md676.

4- Ses vingt-six journaux et dix cahiers de dessins et aquarelles isolées se trouvent dispersés dans différentes collections privées et publiques. La Bibliothèque nationale et universitaire de Strasbourg, la Bibliothèque universitaire de Tübingen et les archives de la Ville de Freudenstadt conservent la plupart des cahiers de dessins ainsi que les aquarelles isolées.

5- Bettelheim, Anton, Biographisches Jahrbuch und Deutscher Nekrolog, Band XVIII (1913), Berlin, 1917, p. 89

6- Pour toutes ces indications concernant la biographie d'Euting, voir Didier, Christophe, « Portrait d'un fondateur : Julius Euting », in La Revue de la BNU, n² 2, 2010, p. 106

7- Graner, Hans , « Julius Euting: Bibliothekar, Forschungsreisender und Orientalist », in Lebensbilder aus Schwaben und Franken, 8. Band, Stuttgart, 1962, p. 309

8- Notz, Hermann, Sechzehnsprachenmännle, Ruhesteinvater und Feuerteufel: Professor Dr. phil. Julius Euting, Freudenstadt, Schwarzwaldverein, 1983, p. 6. Euting avait étudié la peinture à l'eau d'après le livre The English School of Painting in Water-Colours publié en 1861 par le peintre britannique Aaron Edwin Penley (1806-1870) à Londres. Des exemples de ses études se trouvent dans le cahier de peinture « J. E. 18651866 Lahr Oden[wald] », figurant dans la collection privée Weygang-Winter.

9- Graner, Hans, « Julius Euting: Bibliothekar, Forschungsreisender und Orientalist », art. cit., p. 308

10- Graner, Hans, art. cit. Traduction en français des citations originales par l'auteur.

11- Documents divers : dessins de jeunesse, photographies, portraits, fascicule funéraire, fonds Julius Euting, 28 pièces, formats divers, sans lieu, 1830-1913, collection Hans Winter acquise en 2016, MS.7.096, BNU Strasbourg. Lien vers le catalogue en ligne : https://biblio.bnu.fr/opac/resource/fonds-julius-euting-documentsdivers-dessins-de-jeunesse-photographies-portraits-fascicule-funeraire/ $B N U 7839816$ ? sys $b=b n u$. Sur le contexte de leur acquisition, voir Claude Lorentz, « Le fonds Euting s'enrichit », in La Revue de la $B N U, \mathrm{n}^{\circ} 13,2016$, p. 96-98.

12- Graner, Hans, art. cit., p. 314

13- Sur la destruction de la bibliothèque du Temple Neuf suivie de la création de la KULBS, voir la synthèse récente : Barbier, Frédéric (dir.), bibliothèques strasbourg origines-XXI siècle, Paris, Éditions des Cendres / Strasbourg, Bibliothèque nationale et universitaire, 2015.

14- Didier, Christophe, « Portrait d'un fondateur : Julius Euting », art. cit., p. 107 ; idem, « Euting, Julius », in Dictionnaire culturel de Strasbourg (1830-1880), Strasbourg, Presses universitaires de Strasbourg, 2017, p. 184-185. Notons que les fonds anciens des bibliothèques de Strasbourg et de Tübingen sont jusqu'à ce jour classés de la même manière, suivant la " systématique Euting ".

15- Julius Euting fut en 1872 membre fondateur du Club vosgien à Strasbourg et a présidé cette association d'excursions dans la région entre 1876 et 1912. Il s'engageait à faire connaître la beauté naturelle de la région d'Alsace à ses amis allemands et français. À son initiative, une patinoire avait été installée près de l'Orangerie en 1881 par les sapeurs-pompiers de la ville de Strasbourg. Par ailleurs, Euting a aussi donné des cours d'hébreu aux élèves du Gymnase protestant de Strasbourg (cf. Didier, 2010, p. 107).

16- Julius Euting a publié plusieurs guides touristiques comme Les Vosges (avec Curt Mündel), ou encore le Guide illustré de la Ville de Strasbourg et de la Cathédrale (1881), qui a été publié en trois langues.

17- Notz, Hermann, art. cit., p. 18

18- À propos de Charles Huber, voir l'article de Claude Lorentz p. 101.

19- Nöldeke, Theodor, "Julius Euting », in Der Islam: Zeitschrift für Geschichte und Kultur des Islamischen Orients, $\mathrm{n}^{\circ}$ 4, 1913, p. 121-122

20- Ibid. Traduction en français des citations originales par l'auteur.

21- Euting, Julius, Recueil d'aquarelles de paysages arabes, 28 folios avec des illustrations en couleur, dessins au crayon et aquarelles, $250 \times 345 \mathrm{~mm}$, reliure demi-toile et percaline verte, différents lieux, illustrations datées entre 1884 et 1910. Provenance : Hans Winter en 2015. MS.7.094, BNU Strasbourg. Lien vers la notice bibliographique : https://biblio.bnu.fr/opac/resource/ recueil-daquarelles-de-paysages-arabes/BNU7839610?sysb=bnu. Sur le contexte de leur acquisition, voir Claude Lorentz, « Le fonds Euting s'enrichit ", art. cit.

22- Entrée du 23 janvier 1898, journal de Julius Euting lors de l'expédition à Pétra. Conservé et numérisé par la Bibliothèque universitaire de Tübingen. Lien : http://idb.ub.uni-tuebingen.de/ diglit/Md676-11/0007. « Blechrolle » : littéralement « rouleau de tôle ». On peut s'interroger sur ce matériau et son utilité dans une telle expédition.

23- Ehrhard, Albert, « Das griechische Kloster Mâr-Saba in Palästina: seine Geschichte und seine literarischen Denkmäler », in Römische Quartalschrift, 7, 1893, p. 32-79

24- Entrée du 8 février 1898, journal de Julius Euting lors de l'expédition à Pétra. Conservé et numérisé par la Bibliothèque universitaire de Tübingen. Lien : http://idb.ub.uni-tuebingen.de/ diglit/Md676-11/0103.

25- L'aquarelle est une technique picturale fondée principalement sur l'utilisation de trois matériaux de base : de l'eau distillée et mélangée à une gomme arabique, des pigments broyés et agglutinés avec cette même eau, et du papier apte à absorber le liquide et à fixer les pigments sur sa surface.

26- Plusieurs cahiers de peinture de Julius Euting montrent les empreintes de papeteries spécialisées dans l'art graphique, comme « Carl Schleicher \& Schüll » et « Albert Martz ».

27- Entrée du 9 février 1898, journal de Julius Euting lors de l'expédition à Pétra. Conservé et numérisé par la Bibliothèque universitaire de Tübingen. Lien : http://idb.ub.uni-tuebingen.de/ diglit/Md676-11/0105.

28- Roberts, David, The Holy Land, Syria, Idumea, Arabia, Egypt and Nubia from drawings made on the spot by David Roberts...; lithographed by Louis Haghe, London, 1842-1845. L'ouvrage figure dans les collections de la BNU en trois volumes : D.1.450,1; D1.450,2 et D.1.450,3. Une édition en français, plus réduite, en un volume, a été publiée à Bruxelles en 1843. À la BNU sous la cote RF.24.

29- Library of Congress, lien : https://lccn.loc.gov/2002717497. 